Revista científica, arbitrada e indizada, bajo la modalidad electrónica.

Recibido: $08 / 07 / 2020$

Aceptado: 16/07/2020

\title{
LA UNIVERSIDAD CORPORATIVA COMO FUENTE DE APALANCAMIENTO PARA LA ALINEACIÓN DE LA CULTURA ORGANIZACIONAL
}

The corporate university as a source of leverage for the alignment of organizational culture

\author{
Neyersson José Cisneros Garcia \\ UMECIT \\ neyerson-cisneros@hotmail.com \\ Panamá \\ Cira de Pelekais \\ Florida Global University \\ cira.fernandez@floridaglobal.university \\ USA
}

\section{RESUMEN}

El artículo tiene como objetivo analizar la Universidad Corporativa como fuente de apalancamiento para la alineación de la cultura organizacional. Bajo un enfoque positivista, de tipo descriptivo y diseño de campo no experimental. La población conformada por 76 personas. El instrumento utilizado una encuesta tipo Likert con 46 reactivos, validada a través del juicio de expertos y una confiabilidad de 0,872. Los resultados indican que el $100 \%$ de la población encuestada, está de acuerdo con la implementación de la Universidad Corporativa dentro de las organizaciones empresariales, debido a que según sus conocimientos fortalece la cultura organizacional, contribuye de igual forma, a que estas lleguen a un nivel competitivo mayor, respecto a su sector o competencia y el $73,69 \%$ del personal, muestra del estudio, está un poco de acuerdo con las actitudes dentro del área de trabajo, como se dirigen o relacionan entre compañeros.

Palabras clave: Universidad Corporativa, Cultura organizacional, Organizaciones empresariales.

\footnotetext{
ABSTRACT

The article aims to analyze the Corporate University as a source of leverage for the alignment of the organizational culture. Under a positivist approach, descriptive type and non-experimental field design. The population made up of 76 people. The instrument used
} 


\section{Revista científica, arbitrada e indizada, bajo la modalidad electrónica.}

a Likert-type survey with 46 items, validated through expert judgment and a reliability of 0.872. The results indicate that $100 \%$ of the surveyed population agrees with the implementation of the Corporate University within business organizations, because according to their knowledge it strengthens the organizational culture, contributes in the same way, so that they reach a higher competitive level, with respect to their sector or competence and $73.69 \%$ of the staff, sample of the study, somewhat agree with the attitudes within the work area, as they are directed or related between colleagues.

Key words: Corporate University, Organizational culture, Business organizations.

\section{INTRODUCCIÓN}

En la actualidad es cada vez mayor la competitividad en el mundo de los negocios, por lo cual las empresas deben estar actualizadas en cuanto a los agentes diferenciadores, que les permita ser distinguidos dentro de su mercado, es importante la aplicación de estrategias internas y externas que permitan lograr un posicionamiento y a su vez generar una buena rentabilidad.

En este sentido, la investigación que da origen a este artículo, se inicia por la necesidad de analizar la Universidad Corporativa como una herramienta estructurada que sirva de apalancamiento para la cultura organizacional. Tomando como premisa los resultados evidenciados en organizaciones que han incorporado dentro de su estructura, universidades corporativas, con el fin de capacitar, actualizar y adiestrar a su capital humano, logrando de esta manera alinear este recurso con los estándares requeridos, tanto a nivel interno como externo.

Con respecto a lo planteado, al revisar algunas experiencias que existen relacionadas a la temática en estudio, se examina The Walt Disney Company y su Universidad Corporativa llamada Disney University, institución que ha realizado desde el año 1965 importantes aportes en beneficio del capital humano integrante de esta organización, siendo uno de los pioneros en el desarrollo de esta estrategia de gestión.

De igual forma, Disney University ha implementado importantes actividades, propendiendo al fortalecimiento de la cultura de servicio al cliente e innovando con un modelo de negocio en general en sus diferentes rubros de operación, tales como: parques 


\section{Revista científica, arbitrada e indizada, bajo la modalidad electrónica.}

recreativos, división cinematográfica, marcas entretenimiento, estudios, línea de servicios, gestionando eficientemente su Universidad Corporativa, siendo ejemplo de una visión multinacional como una de las compañías de mejores prácticas en la consumación de este tipo de institución al servicio de la organización.

Continuando con la revisión, es posible encontrar al Banco General de Panamá y su Universidad Corporativa BG, fundada en el 2009, evidenciando que en esta institución implementan una fuerte estrategia de alineación de su cultura organizacional apalancada en la escuela de liderazgo, desarrollo personal, así como profesional y cultura organizacional. En este sentido, de acuerdo con Raúl Zubieta, Presidente de Junta Directiva: "La Universidad Corporativa de Banco General, diseña y ejecuta los planes de desarrollo profesional de todos los trabajadores con la alineación fundamental a la cultura de nuestro Banco transformándonos en gente multidisciplinaria y comprometidos con nuestra marca".

Por otro lado, en relación con la cultura organizacional, Ritter (2017), la define como:

El modo en que proceden los participantes de la organización con principio en un conjunto de reconocimientos y valores corporativos, facilitando un marco común de referencia que da una idea semejante del modelo similar del comportamiento ante situaciones específicas. Toda organización tiene cultura corporativa y están llamadas a vivir en un mundo de permanente cambio. (p. 54)

Corroborando lo anterior, Hall (2016), define la cultura organizacional como el motor del cambio en las organizaciones, lo cual alinea a los equipos involucrados hacia un fin común, con valores profundos, arraigados a objetivos claros, esta instancia prepara a las organizaciones para enfrentar el mundo cambiante en el cual se vive, frente a la transformación tecnológica, social, económica además de ambiental.

Asimismo, Conger (2019), indica que la cultura organizacional pretende siempre ir en busca de cambios positivos para las organizaciones, impulsando la creación de nuevas estrategias que permitan alcanzar los objetivos, permitiendo además forjar una identidad propia, con características distintivas impulsando a la transmisión de emociones que se dan bajo la estructura organizacional. 


\section{Revista científica, arbitrada e indizada, bajo la modalidad electrónica.}

En cuanto a lo descrito, Alvarado (2020), representa la cultura como el conglomerado de reglas instructivas que direcciona la conducta de las personas, permitiendo tener características, creencias, comportamientos propios distintivos, arraigados a las personas, siendo este el sistema adecuado para un firme funcionamiento referente a la cultura en las organizaciones.

Con respecto a lo planteado, el artículo tiene como objetivo analizar la Universidad Corporativa como fuente de apalancamiento para la alineación de la cultura organizacional.

\section{BASAMENTO TEÓRICO REFERENCIAL}

La inmersión en los términos asociados contribuye a la expansión de los conocimientos respecto al tema, facilitando la comprensión, al igual proveyendo una guía, sobre la cual se desarrolla este artículo. Por lo cual, se presentan a continuación las bases teóricas:

\section{Universidad Corporativa}

La educación proporciona los conocimientos necesarios para los diferentes contextos a los cuales se enfrenta una persona en la vida diaria; de esta manera este proceso se inicia desde la primera etapa de vida, teniendo en cuenta la sociedad de la cual se rodea, los aspectos, personas que influyen progresivamente en el crecimiento personal; sin embargo, a nivel profesional, esta educación es proporcionada por centros especializados de acuerdo a como el individuo desee formarse o el campo de acción anhelado a realizarse profesionalmente, en este sentido, las empresas también aportan alternativas para propiciar espacios de aprendizaje al individuo, con relación a la función a desempeñar.

Con base en la fundamentación de las Universidades Corporativas, se revisa la teoría plateada por el autor Allen (2002), citado por Vera (2017), en su definición acerca de las universidades corporativas que son:

Entidades educacionales con una herramienta estratégica diseñada para ayudar a la compañía matriz a alcanzar su misión llevando a cabo actividades que cultivan el aprendizaje, conocimiento y sabiduría individual y organizacional. (p.9). 


\section{Revista científica, arbitrada e indizada, bajo la modalidad electrónica.}

En relación con lo planteado, el citado autor señala, que una universidad corporativa tiene completa dependencia a la dirección organizativa; por tanto, debe alcanzar valor de formación continua y a largo plazo, comprometiéndose a la solvencia económica, inclusive en una crisis económica. Con apoyo del domo directivo, es posible observar el impacto al resto de los niveles de la organización, permitiendo tomar decisiones importantes.

Para Ramos (2017), las universidades corporativas tienen completa relación entre los objetivos de formación y los estratégicos de la empresa de forma continua, permitiendo lograr un impacto positivo que con las estructuras tradicionales no se obtiene. En cuanto, a las universidades tradicionales estas dirigen esfuerzos puntuales a la formación profesional y adecuación al cambio, en cambio la UC apunta al desenvolvimiento en el puesto ocupado, planificando el futuro del empleado con visualización de su carrera en concordancia con la organización.

En este sentido, la universidad corporativa se ha venido manejando durante las últimas décadas como una modalidad estratégica de soporte para las organizaciones. Hoy por hoy, las estrategias que impulsan las empresas establecen un desarrollo continuo de competencias y alineación a la cultura organizacional de cada uno de sus colaboradores (Agudelo, 2019).

Parafraseando a Hugas (2017), la universidad corporativa es el vehículo para generar una cultura de aprendizaje, compromiso, retención, atracción y alto desempeño en las organizaciones; permitiendo articular un propósito formativo para el negocio; a su vez contribuir en hacer de la organización un lugar atractivo para trabajar.

Asimismo, Meister (1998), citado por Agudelo (2019), entiende a la UC como una segmentación educacional que opera como la sombrilla estratégica de la organización contribuyendo a la formación de sus colaboradores y de la cadena de valor completa. Por otro lado, Lorenzatti (2000), citado por Agudelo (2019), afirma que es un proceso permanente y orientado a resultados, el cual surge a partir de responder a la pregunta de ¿Qué necesitan aprender los empleados para desempeñarse apropiadamente?. 


\section{Revista científica, arbitrada e indizada, bajo la modalidad electrónica.}

En este sentido, la universidad corporativa se caracteriza por ser un ente sólido, alineado a los objetivos estratégicos del negocio, que involucra a todos los miembros de la organización en sus planes de capacitación y desarrollo, fomentando el crecimiento profesional de cada uno de sus integrantes.

Por lo tanto, la universidad corporativa tendría como herramienta, el diseño de políticas, manuales de procesos, definición de objetivos acordes con los planes estratégicos empresariales, impactando de forma positiva en todas las áreas de la organización, con la finalidad de buscar la calidad laboral para el buen desempeño y la mejora continua de todos los colaboradores.

Reforzado lo anterior, la universidad corporativa propicia la gestión del conocimiento de la empresa, sistematiza el proceso de inducción a la organización, desde la llegada del nuevo miembro, acompañándolo en su inserción a la ruta de su camino laboral. Velando por su estabilidad interna, retención del talento, aportando al buen clima organizacional y formando talentos preparados para desempeñarse con estándares altos.

Las conceptualizaciones anteriores permiten comprender el significado de la Universidad Corporativa como una estrategia a través de la cual se puedan alcanzar los objetivos de las empresas en rápido crecimiento, unificando todos los niveles de la misma, contribuyendo así al alcance de las misiones, objetivos, metas directivas y toma de decisiones cruciales para el desarrollo.

\section{Funciones de la Universidad Corporativa}

De acuerdo con Allen (2002), citado por Vera (2017), una Universidad Corporativa mantiene funciones variantes en cuanto a las diferentes empresas o tiempo; sin embargo, se destacan las principales: la académica como soporte para la formación del capital humano, de investigación, al igual de desarrollo como columna de capital intelectual, de extensión o difusión sustentada en las alianzas de compromiso social.

\section{Objetivos y pasos para la creación de una Universidad Corporativa}

La creación de una Universidad Corporativa tiene como objetivo principal evolucionar hacia un patrón de capacitación con desarrollo de gran trascendencia 


\section{Revista científica, arbitrada e indizada, bajo la modalidad electrónica.}

involucrando a la totalidad de los miembros de una organización. Por lo cual, resulta indispensable tener claro los objetivos a lograr, tales como: ser un instrumento para la notoriedad, prestigio de la empresa de su oferta educativa tanto interna como externa, convertir el aprendizaje en un proceso unificador corporativo geográficamente facilitando la transmisión de valores, asegurando una formación alineada con las competencias requeridas para la evolución profesional de sus miembros. (Ramos, 2016, p.30).

\section{Proceso de la Universidad Corporativa}

En primera instancia, para iniciar el proceso de la universidad corporativa se deben incluir pilares de sostén para la gestión estratégica, estos son la visión con el objetivo de precisar a donde se quiere llegar; una segunda etapa es la definición de los valores, los cuales brindan la orientación en la visión; un tercer pilar corresponde a la misión el cual conlleva a saber el enfoque de ¿Para quién hacerlo?, ¿Cómo hacerlo?. La construcción de estos en relación con la identidad del negocio y su estrategia corporativa base misión, visión y valores de la organización. (Allen, 2002, p. 9, citado por Vera, 2017)

\section{Estrategia de la Universidad Corporativa}

La estrategia de la UC según Ramos (2017, p. 15), expresa que "la estrategia despliega los principales recursos y esfuerzos para alcanzar sus objetivos, un curso de acción consistentemente deseado y determinado de forma anticipada, con la finalidad de asegurar el logro de los objetivos de la empresa"

\section{Organización interna de la Universidad Corporativa}

Es de vital importancia que se identifique en primer lugar, lo que necesita realmente la empresa para llevar a cabo una Universidad Corporativa con el fin de mejorar el desempeño individual, de equipo y organizacional.

Ramos (2017), establece que para ello se pueden basar en el Sistema de Detección de Necesidades Multinivel (SDNM), instrumento mediante el cual se busca convertir los requerimientos del negocio en iniciativas a realizar en la UC, profundizando en cómo realizar, desde un enfoque proactivo, el análisis de las necesidades actuales o futuras de la empresa en tres niveles: estratégico, táctico además de operativo. 
Revista científica, arbitrada e indizada, bajo la modalidad electrónica.

\section{Situación actual de las compañías que cuentan con Universidad Corporativa}

Acosta (2015), en su artículo para la revista digital Expok menciona 10 de las empresas más reconocidas a nivel mundial, las cuales cuentan con Universidad Corporativa, estas son:

1. Apple: para esta empresa su creador Steve Jobs estableció la Universidad Apple cuyo programa de formación interno se encarga de enseñar la visión y prácticas para seleccionar nuevos empleados. En los temas que además incorporaron en esta universidad van desde el diseño o comunicación hasta gestión empresarial, los profesores involucrados en su mayoría son graduados de Harvard y Stanford. (Acosta, 2015).

2. Mc Donald's: según lo expresado por Acosta (2015), esta compañía creó hace más de 50 años la Hamburger University (Universidad de Hamburguesa) en Estados Unidos, persiguiendo la meta de establecer un campus educativo que instruya a los nuevos talentos acerca de todo lo concerniente al servicio que se debe de proporcionar a los clientes, así como las claves para una eficiente higiene en los restaurantes.

3. Pepsi: para esta compañía PepsiCo University es solo uno de los programas a través del cual la empresa busca capacitar y entrenar a todos los talentos a colaborar con la marca a partir de cursos que incluyen diversidad de temas como finanzas, liderazgo, retroalimentación y desarrollo estratégico.

4. Volkswagen: instaura la UC con la intención de servir a la sociedad y a la corporación empresarial, la práctica y el discernimiento de la organización Volkswagen en materia técnica, de gestión y de desarrollo humano. (Acosta, 2015).

5. Coca - Cola: la empresa desarrolla capacitación en liderazgo utilizando como capacitación un $70 \%$ en el trabajo, un $20 \%$ por coaching y un $10 \%$ en entrenamiento. Una décima parte de sus empleados son forasteros, y la 


\section{Revista científica, arbitrada e indizada, bajo la modalidad electrónica.}

compañía brinda oportunidades de experimentar en la arena global. (Acosta, 2015).

6. Pixar: Su misión ha sido siempre formar a los mejores, una labor tomada muy en serio, la cual ha ayudado a convertir a los estudios en todo un gigante del mundo del entretenimiento. (Acosta, 2015).

7. Conde Nast: la editorial de revistas internacional, creada en el 1907 por Condé Montrose Nast. Su U.C capacita a sus estudiantes de modo que puedan laborar en la totalidad de las áreas existente de manera emocionante e innovadora. (Acosta, 2015).

8. General Motors: la Universidad de Kettering ofrece 14 licenciaturas, 5 títulos de postgrado, y concentraciones a menores de edad que consienten ceder una educación teórica y práctica mejor que cualquier otra institución en el mundo. (Acosta, 2015).

9. Disney: Van France, el primer ejecutivo fundó la Universidad Disney para dar lección a los empleados cómo "crear felicidad", por lo cual, los miembros de la compañía asimilan todos los detalles de amabilidad, pulcritud y seguridad necesarios para hacer de los parques temáticos una experiencia única. (Acosta, 2015).

10. Cinemex: Universidad Cinemex también ofrece capacitación a los talentos permitiéndoles involucrarse de maneras más sólida con la misión y visión de la empresa. (Acosta, 2015).

La implementación de Universidades Corporativas a estas empresas les ha permitido garantizar la educación continua correcta y oportuna, adicionalmente, esa formación es coherente con los objetivos corporativos.

De esta manera, han podido optimizar los recursos, invirtiendo en un aprendizaje realmente impactante en la actividad de la organización y aumentando la competitividad. El aprendizaje comienza a formar parte esencial de estas organizaciones, apuntando hacia 


\section{Revista científica, arbitrada e indizada, bajo la modalidad electrónica.}

aquellos conocimientos, habilidades y actitudes fundamentales permitiendo a todo el personal contribuir al logro de los planes estratégicos institucionales.

En este sentido, las empresas con universidad corporativa consienten instituir una relación entre sus estrategias internas y los aprendizajes de esta manera trazar un camino para el mejoramiento, con beneficios de posicionamiento, alcance de la misión, entre otros.

En Panamá, se menciona a la empresa con renombre: Banco General, institución financiera con formación educativa a través de su Universidad Corporativa, ofrece instrucción académica de alta calidad, con amplia infraestructura dando así el soporte físico adecuado, equipo tecnológico de última generación, personal altamente calificado producto del enlazamiento con acreditadas universidades nacionales e internacionales.

\section{Programas de formación en las universidades corporativas}

En cuanto a la duración de los programas inmersos en UC, estos no son como los estudios de Grado en universidades tradicionales, el objetivo principal es enlazar la formación con la visión empresarial, por lo cual se hace mayor semejanza a los estudios de tipo maestría u otros estudios a nivel de posgrado, inclusive se hace relevante mencionar que varias UC han logrado convalidar determinados programas de estos tipos. (Gómez, 2018).

Otro aspecto, mencionado por el citado autor, es la manifestación de las nuevas tecnologías, contribuye en la forma de impartir los conocimientos. Aun cuando una clase impartida de forma online sea más barata porque evita los traslados y, por lo tanto, evita bajadas en la productividad, las compañías deben apuntar en cuanto les sea posible a la formación telemática, de esta manera, se mejorarán los resultados, una empresa privada tiene como objetivo principal el lucro para su sustentabilidad a largo plazo.

A su vez, es importante mostrar la diversidad en asignaturas a impartir, y la dependencia relacionada en cuanto a las necesidades de las compañías. Por lo cual, pueden ser atención al cliente, buenas prácticas, riesgos laborales, tendencias de moda, nuevos productos financieros, responsabilidad social, entre otros. La superioridad de este tipo de estudios es la flexibilidad, además de adaptación a las necesidades. (Gómez, 2018). 


\section{Revista científica, arbitrada e indizada, bajo la modalidad electrónica.}

\section{Cultura organizacional}

Núñez (2009), citado por Falcones (2014), indica:

La cultura deberá pensarse como el proceso de asimilación, producción, difusión, asentamiento de ideas y valores en que se funda la sociedad, es el conjunto de representaciones colectivas, creencias, usos del lenguaje, difusión de tradiciones y estilos de pensamiento que articula la conciencia social, es el ámbito en que se producen y reproducen nuestras formas de vida e ideología; visto así la cultura es un mecanismo de regulación social. (p. 3).

Parafraseando a Núñez (2009), la cultura es conocida como las características que denotan a los miembros de un país, las cuales no se desprenden de estas, inclusive permiten que estos se sientan identificados en especial cuando están en otros lugares lejos de su país.

En este sentido, el concepto de cultura en las organizaciones se refiere a "el sistema de significados pública y colectivamente aceptados, operando para un grupo determinado en un tiempo dado". (Cujar et al., 2013, p. 351).

Para obtener una mejor perspectiva de ello, se hace referencia en lo expuesto por Robbins (2009), citado por Falcones (2014), quien sostiene que la cultura organizacional está estrechamente relacionada a la unificación entre la cultura individual y las grupales inmersas en el interior de una organización.

Los dos enfoques coinciden en que constituye un distintivo propio de cada organización a través de las características particulares adoptadas por los empleados que incide en ser diferenciador. Por lo cual, la cultura organizacional se muestra en particular en la relación existente y conductas propias con los grupos de interés, siendo uno de ellos el cliente.

Por otro lado, en relación con la cultura organizacional y su papel en las empresas modernas, González (2019), la define como:

El modo en que proceden los participantes de la organización con principio en un conjunto de reconocimientos y valores corporativos, facilitando un marco común de referencia que da una idea semejante del modelo similar del comportamiento ante situaciones específicas. Los 


\section{Revista científica, arbitrada e indizada, bajo la modalidad electrónica.}

fundadores de la empresa ejercen una fuerte influencia en la formación de su cultura.

Corroborando lo anterior, Infer y Infer (2018), define la cultura organizacional como el punto de partida del cambio en las organizaciones, lo cual alinea a los equipos involucrados hacia un fin común, con valores profundos, arraigados a objetivos claros, esta instancia prepara a las organizaciones para enfrentar el mundo cambiante en el cual se vive, frente a la transformación tecnológica, social, económica además de ambiental.

Asimismo, Dueñas (2016), indica que la cultura organizacional pretende siempre ir en busca de cambios positivos para las organizaciones, impulsando la creación de nuevas estrategias que permitan alcanzar los objetivos, permitiendo además forjar una identidad propia, con características distintivas impulsando a la transmisión de emociones que se dan bajo la estructura organizacional.

\section{Características de la cultura organizacional}

Las características que representan la cultura organizacional están determinadas de la siguiente manera, según Robbins (1993), citado por Falcones (2014, p. 9):

1. Identificación de los miembros: Se refiere a los empleados de las organizaciones.

2. Fortaleza grupal: los esfuerzos contribuyen al bien de todos.

3. Orientación hacia las personas. Decisiones en base a las consecuencias que estas pueden tener en los miembros de la organización.

4. Unificación de las áreas: Operación de forma independiente, pero a su vez armónica.

5. El control: De la conducta de los miembros de la organización.

6. La flexibilidad al riesgo.

7. Los principios de remuneración. Asignación de recompensas, aumento en los pagos de salario, promociones, en conformidad con la productividad, por su antigüedad, las preferencias, u otros factores. 


\section{Revista científica, arbitrada e indizada, bajo la modalidad electrónica.}

8. La forma hacia el logro de metas o de los medios.

9. La orientación hacia un sistema abierto: manejo de cambios externos.

10. Comprensión del conflicto: manejo de conflictos.

\section{Factores que influyen en la cultura organizacional}

En este sentido, según Robbins (1993) Falcones (2014, p. 6), entre los factores que influyen en la formación de la cultura organizacional están:

- Factores organizacionales:

- Factores circunstanciales: como regulaciones vigentes que permitan llevar a cabo o no una función.

- Factores físicos: como la ubicación, accesibilidad, nivel y calidad de vida del medio.

- Factores geográficos: La ubicación geográfica de la organización también influye en la cultura organizacional.

- Factores del entorno

- Escenario físico: se refiere al transporte, alimentación, entre otros, ofrecidos por la organización al trabajador.

\section{MATERIALES Y MÉTODOS}

Al comprender que el objetivo del artículo es analizar la Universidad Corporativa como fuente de apalancamiento para la alineación de la cultura organizacional, se plantea una investigación positivista cuantitativa. Desarrollada bajo un tipo descriptivo, con un diseño de campo no experimental, según lo señala Pelekais et al., (2015).

Para la selección de la muestra, se tomó como referencia a los autores Otzen y Manterola (2017), los cuales establecen que este tipo de muestreo "admite elegir aquellos casos accesibles que accedan ser incluidos". El muestreo probabilístico incluye la capacidad que tiene cada involucrado en la población de integrar la muestra.

La muestra del estudio queda determinada por un total de 76 personas, dadas como resultado luego de la aplicación de la fórmula preestablecida. Esta cantidad de personas son las que forman parte de las encuestas y colaboran en cuanto a la recolección de la información necesaria para el alcance del objetivo propuesto. 


\section{Revista científica, arbitrada e indizada, bajo la modalidad electrónica.}

La encuesta utilizada como técnica permite conseguir información que suministra un grupo o muestra de sujetos acerca de si mismos, o en relación con un tema en particular. (Arias, 2016, p. 70).

En cuanto a confiabilidad del estudio, se aplicó una prueba piloto que permitió la obtención de datos confiables para el instrumento. En este sentido, según Hernández et al., (2018, p. 42), "La prueba piloto se realiza con una pequeña muestra inferior a la definitiva, ubicada fuera de la población"

Asimismo, la visualización de los datos fue considerada de acuerdo a los expresado por Urribarri et al., (2014), citado por Chuacanes y Suárez, (2015, p. 48), para quienes es una de las técnicas de análisis más demandada y apreciada, por lo fácil que resulta a través de un gráfico o imagen detectar patrones en los datos. Es especialmente útil cuando se busca entender grandes volúmenes de datos de forma rápida y simplificada.

\section{RESULTADOS}

Los resultados indican que el 100\% de la población encuestada, está de acuerdo con la implementación de la Universidad Corporativa dentro de las organizaciones empresariales, debido a que según sus conocimientos fortalece la cultura organizacional, contribuye de igual forma, a que estas lleguen a un nivel competitivo mayor, respecto a su sector o competencia y el 73,69\% del personal, muestra del estudio, está un poco de acuerdo con las actitudes dentro del área de trabajo, como se dirigen o relacionan entre compañeros.

Estos resultados coinciden con lo expresado por Ramos (2017), quien relaciona los objetivos de formación con los estratégicos de la empresa, para lo cual se obtuvo un 100\% de totalidad en acuerdo que realmente si se está relacionado y contribuyen mutuamente para el alcance de los objetivos organizacionales.

De igual manera, el 73,69\% del personal, muestra del estudio, está un poco de acuerdo con las actitudes dentro del área de trabajo, como se dirigen o relacionan entre compañeros, mientras que el 19,74\% está totalmente de acuerdo y el 6,57\% no está de acuerdo. 


\section{Revista científica, arbitrada e indizada, bajo la modalidad electrónica.}

Asimismo, queda confirmada la teoría establecida por Allen (2002) citado por Vera (2017), coincidiendo con los resultados encontrados respecto a las Universidades Corporativas, indicando que las UC "son herramientas estratégicas diseñadas para ayudar a la compañía matriz a alcanzar su misión” por lo que es así, las compañías logran un impacto positivo a través de las UC, reflejado en la sabiduría individual y organizacional.

Esto permite hacer referencia a lo planteado por Wilkins and Ouchi, (1983), citado por Falcones (2014), quienes señalan que la cultura organizacional "se establece a partir de como los miembros de la organización desarrollan colectividad a través de las experiencias compartidas", por lo que es tan importante que el comportamiento entre los trabajadores sea siempre de excelencia.

Con respecto al desempeño en procesos, para la respuesta totalmente de acuerdo, se obtuvo un alto porcentaje de coincidencia, lo cual se refiere a que los empleados realmente son responsables en la realización de los trabajos, desde completar las tareas asignadas, organización, autosupervisión, proactividad, entre otros. Aun cuando es positivo que exista este alto índice de empleados siempre se debe considerar el otro porcentaje que no estuvo totalmente de acuerdo y mejorarlo a través de la universidad corporativa, al igual que la fortaleza grupal, la unificación de áreas y controles internos.

En cuanto a los factores físicos, la empresa SmartBrix, mantiene espacios que contribuyen a la realización de las tareas con completa comodidad, permitiendo la movilidad necesaria y la calidad de vida en la misma. Con respecto a los factores del entorno, también contribuyen favorablemente, debido a que cuentan con rutas de traslado, tiempos y espacios para la alimentación y asistencia médica de emergencia.

\section{CONCLUSIONES}

Los encuestados concuerdan en un $100 \%$, que son muchas las ventajas obtenidas para las organizaciones empresariales que poseen Universidades Corporativas, las cuales pueden unificar los objetivos de la empresa con los de la educación y lograr un mayor compromiso por parte de sus empleados mediante la instrucción en Universidades Corporativas con programas combinados, es decir, de modalidad virtual y presencial. 


\section{Revista científica, arbitrada e indizada, bajo la modalidad electrónica.}

Por otro lado, una vez procesados los datos, mediante la visualización de los mismos, con respecto a la variable Universidad Corporativa, se obtiene el favorecimiento de la aplicación de esta estrategia debido a los resultados positivos presentes en compañías internacionales y nacionales que hacen uso de este tipo de instituciones, las cuales le han beneficiado en su cultura organizacional y nivel de competitividad.

Para el caso de la variable, Cultura Organizacional, el proceso de gestión interna está relacionado al comportamiento organizacional, en el cual se deben mejorar las actitudes entre compañeros, factor que dio una frecuencia un poco baja en comparación a otros aspectos como la conducta respecto a la asignación de tareas, puntualidad, honestidad y responsabilidad, para los cuales fue mayor el porcentaje de indicación.

Finalmente, la empresa SmartBrix, se caracteriza por poseer una cultura organizacional que requiere de reforzamientos y mejoras para una óptima operación de sus colaboradores, siendo la implementación de una U.C la mejor estrategia que deben ejecutar.

Con respecto a las recomendaciones, se les sugiere seguir el ejemplo que brindan las otras empresas nacionales e internacionales, en cuanto a la implementación de U.C, las mismas que muestran resultados positivos, elevando su nivel de competitividad. Esto se hace a través de la observación y reuniones de asesoramiento en acuerdo con estas empresas para recibir su opinión.

\section{REFERENCIAS BIBLIOGRÁFICAS}

Acosta, C. (2015). 10 empresas con universidades en las que querrás estudiar. Expox. Recuperado de: https://www.expoknews.com/10-empresas-con-universidades-en-lasque-querras-estudiar/

Agudelo, R. (2019). Universidades Corporativas: la importancia de crear una cultura de capacitación empresarial. Recuperado de: http://www.elogos.cl/universidadescorporativas-la-importancia-crear-una-cultura-capacitacion-empresarial/

Alvarado, O. (2020). El real poder de la cultura organizacional. Monterrey, México. Recuperado de: https://www.elfinanciero.com.mx/monterrey/ oscar-alvarado -el-realpoder-de-la-cultura-organizacional.

Arias, F. (2016). El proyecto de investigación: Introducción a la metodología científica. Caracas, Venezuela. Editorial: Episteme. $7^{\circ}$ Edición.

Cújar, A. Ramos, C. Hernández, H. López, J. (2013). Cultura Organizacional: Evolución en la medición. (Estudios gerenciales). Recuperado de: 


\section{Revista científica, arbitrada e indizada, bajo la modalidad electrónica.}

https://www.researchgate.net/publication/273123649_Cultura_organizacional_evoluc ion en la medicion

Dueñas, F . (2016). 8 pasos para gestionar el cambio de la cultura organizacional . Recuperado de : https://blog.acsendo.com/8-pasos-gestionar-cambio-la-culturaorganizacional-2/

Falcones, J. (2014) Estudio descriptivo de la cultura organizacional de los colaboradores de la unidad de negocio de SUPPLY CHAIN Guayaquil de Nestlé Ecuador. Universidad de Guayaquil. (Tesis maestría) Recuperado de: http://repositorio.ug.edu.ec/bitstream/redug/6287/1/Tesis\%20Cultura\%20organizacio nal.pdf

Gómez, J. (2018). Aprendizaje: Las Universidades Corporativas. Cerem. Recuperado de: https://www.cerembs.co/blog/aprendizaje-las-universidades-corporativas

González S., F. (2019). La cultura organizacional y su papel dentro de la empresa moderna. Recuperado de https://www.gestiopolis.com/la-cultura-organizacional-y-supapel-dentro-de-la-empresa-moderna/

Hernández S., Mendoza C. (2018). Metodología de la investigación: las rutas cuantitativa, cualitativa y mixta. México: McGraw Hill.

Infer, G., Infer, H. (2018). El Cambio personal es el motor del cambio en las organizaciones. (Articulo) Recuperado de: https://www.transform-action.net/elcambio-personal-es-el-motor-del-cambio-en-las-organizaciones/

Hugas, J. (2017). Universidades Corporativas: alineando personas y estrategia. Recuperado de:

https://www.researchgate.net/publication/314403644_Universidades_Corporativas_al ineando_personas_y_estrategia

Otzen, T., Manterola, C. (2017).Técnicas de muestreo sobre una población a estudio. Int. J. Morphol., 35(1):227-232.

Pelekais, C; El Kadi, O; Seijo, C; Neuman, N. (2015). El ABC de la investigación: Una aproximación teórico-práctica. Ediciones Astro Data. Venezuela.

Ramos, T. (2016), Universidades corporativas: Forjando personas para ganar el futuro. Editorial Universitat Oberta de Catalunya UOC. España

Ritter, M. (2017). Cultura organizacional. Disponible en: https://vizion360.xyz/librocultura-organizacional-de-michael-ritter/

Vera, S. (2017), Universidad Corporativa: Una comparativa con la escuela de negocios (ICADE Business School - Repsol, Madrid. (Tesis maestría) Recuperado de: https://repositorio.comillas.edu/xmlui/bitstream/handle/11531/24134/TFM000757.PD F? sequence $=1 \&$ isAllowed $=\mathrm{y}$ 